\title{
A HANDLEBODY WITH ONE PILLBOX HAS NO FAKE 3-CELLS
}

\section{J. O. BERGE AND MATTHEW G. BRIN}

ABSTRACT. The main result establishes upper bounds on the rank of $H_{1}$ of orientable 3-manifolds with fake 3-cells and on the rank of $\pi_{1}$ of closed orientable 3-manifolds with fake 3-cells. As a consequence we get that a 3-manifold obtained by sewing one pillbox on a handlebody of arbitrary genus cannot contain a fake 3-cell.

1. Introduction. The Poincaré conjecture, an open question of long standing in the topology of 3-manifolds, states that any compact 3-manifold without boundary that is simply connected must be a 3-sphere. An equivalent statement is that a compact, simply connected 3-manifold with connected, 2-sphere boundary is a 3-cell. A counterexample to the second statement is called a fake 3-cell. Since any orientable 3-manifold may be obtained by sewing pillboxes onto a handlebody of sufficient genus, it is of interest to know which objects of this type cannot contain a fake 3-cell. C. D. Feustel has shown in [1] that if a handlebody with one pillbox has free fundamental group, then the resulting manifold is also a handlebody, and thus does not contain a fake 3-cell. We show that sewing a single pillbox onto a handlebody in any fashion gives a manifold that contains no fake 3-cells. This is obtained as a corollary to Theorem 3 below. This theorem is based on the fact that lens spaces contain no fake 3-cells, and on a result of Haken that allows us to induct from there. A restatement of a weakened form of Haken's theorem [3, Theorem, p. 84] is as follows:

Let a closed orientable 3-manifold $M$ be the union of two handlebodies $H_{1}$ and $H_{2}$ whose intersection $T$ is their common boundary. Suppose $M$ contains a 2-sphere that bounds no 3-cell. Then $M$ contains a 2 -sphere $S$ that bounds no 3-cell and so that $S \cap T$ is a simple closed curve.

In the following we will work in the category of simplicial complexes and peicewise linear maps. All manifolds are compact and orientable, all homologies will be over $Z$, the rank of homology groups will be the free rank and the rank of homotopy groups will be the minimal number of generators needed for a presentation. A closed manifold will be compact and without boundary. By a Heegaard splitting of genus $n$ we will mean two 3-dimensional handlebodies of genus $n$ sewn together along their boundaries by a

Received by the editors July 3, 1974 .

AMS (MOS) subject classifications (1970). Primary 55A40, 57A10; Secondary 57A65.

Key words and phrases. 3-manifold, homotopy 3-sphere, Heegaard splitting, handlebody, first homology, fundamental group. 
homeomorphism. All closed, orientable 3-manifolds can be represented as a Heegaard splitting of genus $n$ for some $n$. If $X$ is a closed, orientable 3manifold, then the Heegaard genus of $X$ is the smallest integer $n$ so that $X$ admits a Heegaard splitting of genus $n$. A pillbox will be a 3-cell regarded as the cross product of a 2 -cell, $D^{2}$, and the unit interval, $I$. A 3-manifold $X$ will be called a handlebody with pillboxes if it is obtained be sewing a finite number of pillboxes onto a handlebody $H$ by homeomorphisms identifying the annuli $\partial D^{2} \times I$ with disjoint annuli contained in $\partial H$. The genus of such an $X$ will be the genus of $H$. If $X$ is a handlebody with pillboxes, of genus $n$, or a closed 3-manifold of Heegaard genus $n$, then the homology deficiency of $X$ will be $n-\operatorname{rank}\left[H_{1}(X)\right]$; the homotopy deficiency will be $n-\operatorname{rank}\left[\pi_{1}(X)\right]$. Note that both numbers are nonnegative and that rank $\pi_{1}(X) \geq \operatorname{rank} H_{1}(X)$.

2. Lemma 1. Let $X$ be a compact, orientable 3-manifold with connected boundary of genus $g$. Then

(a) rank $H_{1}(X) \geq g$,

(b) there is a handlebody $H$ of genus $g$ and a homeomorphism $h: \partial X \rightarrow$ $\partial H$ so that rank $H_{1}\left(X \cup_{h} H\right)=\operatorname{rank} H_{1}(X)$.

Proof. For orientable manifolds we know, for homology over $Z$, that $2 g=\operatorname{rank} H_{1}(\partial X)=$ twice the rank of [Image $i_{*}: H_{1}(\partial X) \rightarrow H_{1}(X)$ ] so $(a)$ is true. Let $j_{*}$ be the projection from [Image $i_{*}$ ] onto its free part. $j_{*} i_{*}$ is a map from a free abelian group of rank $2 g$ onto a free abelian group of rank $g$ and we have $H_{1}(\partial X) \cong \operatorname{Ker}\left(j_{*} i_{*}\right) \oplus \operatorname{Im}\left(j_{*} i_{*}\right)$.

Let $\alpha$ be a primitive element in $\operatorname{Ker}\left(j_{*} i_{*}\right)$. $\alpha$ can be represented by a simple closed curve, $J$, on $\partial X$ that does not separate $\partial X[5$, Proposition 2.11]. If we obtain a new manifold $\widetilde{X}$ by sewing a pillbox onto $X$ along a regular neighborhood of $J$ in $\partial X$, we will kill $i_{*}(\alpha)$. However $i_{*}(\alpha)$ was either zero or torsion so that rank $H_{1}(\tilde{X})=\operatorname{rank} H_{1}(X)$. Since $\partial \tilde{X}$ is connected and of smaller genus than $\partial X$ we have (b) by induction.

Corollary 2. Let $X$ be a handlebody $H$ with pillboxes such that $\partial X$ is connected. Then $X$ can be embedded in a closed 3-manifold $M$ with Heegaard splitting $(H, \overline{M-H})$ such that homology deficiency of $M=$ bomology deficiency of $X$.

Theorem 3. Let $X$ be a handlebody $H$ with pillboxes or a closed 3-manifold with Heegaard splitting $(H, \overline{X-H})$. If $X$ contains a fake 3-cell, then the homology deficiency of $X$ is at least 2.

Proof.

Case 1. $X$ is closed or $\partial X=S^{2}$. We can assume that $X$ is closed since we can always sew a 3-cell onto $\partial X$. Let $C$ be a fake 3-cell in $X$ and let $D=\overline{X-C}$. Since lens spaces contain no fake cells we can assume that genus $X \geq 2$. If $X$ is a homotopy 3 -sphere, genus $X \geq 2$ and the desired 
result follows; hence we may suppose that

$$
1 \neq \pi_{1}(X)=\pi_{1}(C) * \pi_{1}(D)=\pi_{1}(D) .
$$

Thus $\partial C$ is a 2 -sphere that does not bound a 3 -cell. Since genus $X \geq 2$ we can use Haken's theorem to find a 2 -sphere $S$ in $X$ so that $S \cap H$ and $S \cap \overline{(X-H)}$ are properly embedded disks separating each of $H$ and $\overline{X-H}$ into two handlebodies of nonzero genus. If genus $X=2$ this gives a connected sum of lens spaces which contradicts the hypothesis, so $\pi_{1}(X)=1$. If genus $X>2$ let the complementary domains of $S$ be $M_{1}$ and $M_{2}$. It is a consequence of Milnor's prime decomposition theorem [4] that either $M_{1}$ or $M_{2}$ contains a fake 3-cell and by induction has homology deficiency at least 2 (see also [2, Theorem 2]). $H_{1}(X)=H_{1}\left(M_{1}\right) \oplus H_{1}\left(M_{2}\right)$ and the theorem follows.

Case 2. $\partial X$ is connected. Follows from Corollary 2 and Case 1.

Case 3. $\partial X$ is not connected. We will connect up $\partial X$ by adding 1-handles to $H$ along pairs of disks disjoint from the annuli that $\partial H$ shares with the pillboxes. Each 1-handle will be sewn to $\partial X \cap \partial H$ along a pair of disks in different components of $\partial X$, and enough 1-handles will be added so that the resulting manifold has connected boundary. Since each handle increases genus $H$ by one and rank $H_{1}(X)$ by one, the deficiency is the same and we are reduced to Case 2. This completes the proof.

The proper substitutions in the proof of Case 1, replacing homology by homotopy, $H_{1}$ by $\pi_{1}$, and direct product by free product, will give:

Theorem 4. Let a closed orientable 3-manifold M have a fake 3-cell. Then the homotopy deficiency of $M$ is $\geq 2$.

Corollary 5. Let $X$ be a handlebody plus one pillbox. Then $X$ contains no fake 3-cells.

Proof. Lemma 1(a) and Theorem 3.

Note. If $m$ is the smallest integer such that a homotopy 3-sphere exists of Heegaard genus $m$, then Theorems 3 and 4 can be restated with deficiency $m$, and Corollary 5 can be restated with $(m-1)$ pillboxes.

\section{REFERENCES}

1. C. D. Feustel, On pasting balls to handle-bodies, Bull. Amer. Math. Soc. 76 (1970), 720-722. MR 41 \#4517.

2. Jonathan L. Gross, Manifolds in which the Poincaré conjecture is true, Trans. Amer. Math. Soc. 142 (1969), 177-189. MR 39 \#7606.

3. W. Haken, Some results on surfaces in 3-manifolds, Studies in Modern Topology, Math. Assoc. Amer. (distributed by Prentice-Hall, Englewood Cliffs, N. J.), 1968, pp.39-98. MR 36 \#7118.

4. J. Milnor, A unique decomposition theorem for 3-manifolds, Amer. J. Math. 84 (1962), 1-7. MR 25 \#5518.

5. Jose Vrabec, Submanifolds of acyclic 3-manifolds, Pacific J. Math. 49 (1973), 243-263.

DEPARTMENT OF MATHEMATICS, UNIVERSITY OF WISCONSIN, MADISON, WISCONSIN 53706 